\title{
Role of the DECAF Score in Predicting In- hospital Mortality in Acute Exacerbation of Chronic Obstructive Pulmonary Disease
}

\author{
Mubeen A. Memon ${ }^{1}$, Sheeba Faryal ${ }^{1}$, Naveed Brohi ${ }^{2}$, Besham Kumar ${ }^{3}$ \\ 1. Pulmonology, Civil Hospital, Jamshoro, PAK 2. Intenal Medicine, Civil Hospital, Jamshoro, PAK 3. \\ Internal Medicine, Jinnah Postgraduate Medical Center, Karachi, PAK
}

$\square$ Corresponding author: Besham Kumar, beshamkumar916@gmail.com Disclosures can be found in Additional Information at the end of the article

\section{Abstract}

\section{Introduction}

Acute exacerbations of chronic obstructive pulmonary disease (AECOPD) can be fatal. In 2012, a comprehensive score was developed to predict the risk of in-hospital mortality in AECOPD called the dyspnoea, eosinopenia, consolidation, acidemia, and atrial fibrillation (DECAF) score. We conducted this study to assess the value of the DECAF score as a clinical prediction tool that claims to stratify all patients with AECOPD by risk accurately.

\section{Methods}

We conducted a prospective study of patients admitted to the intensive care unit (ICU) of the Department of Pulmonology in Civil Hospital, Jamshoro, from January 2016 to December 2018. Our inclusion criteria were that the patient must be age 35 years or older, have a primary clinical diagnosis of AECOPD, spirometry consistent with airflow obstruction, and have a smoking history of $\geqslant 10$ cigarette pack per year. We excluded patients who had domiciliary ventilation, survival-limiting comorbidities (such as metastatic malignancy), and a primary reason for admission other than AECOPD. All sociodemographic data were collected at the time of admission, including age, gender, co-morbidities, housebound status, and number of previous AECOPD. Clinical data collected included plain chest x-ray, spirometry, electrocardiogram, arterial blood gases analysis, complete blood count, kidney function test, liver function test, and serum electrolytes. A DECAF score was applied to each patient. We noted in-hospital mortality and compared the characteristics of survivors and non-survivors. Data were analyzed using IBM SPSS for Windows, version 19.0 (IBM Corp, Armonk, NY).

\section{Results}

Received 05/17/2019 Review began 05/18/2019 Review ended 05/19/2019 Published 06/04/2019

\section{(c) Copyright 2019}

Memon et al. This is an open access article distributed under the terms of the Creative Commons Attribution License CC-BY 3.0., which permits unrestricted use, distribution, and reproduction in any medium, provided the original author and source are credited.
A total of 162 patients were included in the study. The mortality rate was $13 \%(n=21)$. More survivors had a DECAF score from zero to three than non-survivors. The difference in the number of survivors vs. non-survivors was statistically significant for DECAF scores zero and one. For DECAF scores four and five, there were more patients in the "non-survivors" group, and the differences were statistically significant. None of the patients scored six on DECAF.

\section{Conclusion}

Patients with a DECAF score of four or higher have a significant risk of mortality. DECAF is a simple tool that predicts mortality that incorporates routinely available indices to stratify AECOPD patients into mortality risk categories. 
Categories: Internal Medicine, Pulmonology

Keywords: predictors of mortality, in-hospital mortality, copd, copd exacerbation, acute exacerbation of copd, decaf score

\section{Introduction}

Chronic obstructive pulmonary disease (COPD) is among the more common noncommunicable diseases in the field of pulmonology. It is characterized by limited airflow and has a debilitating impact on both quality of life and life expectancy [1]. COPD is the third-leading cause of death [2]. COPD has a chronic, stable pattern aggravated by sudden, acute episodes of dyspnea and productive cough. These acute exacerbations of COPD (AECOPD) may be triggered by tobacco smoking or air pollution [2] and can be fatal [3]. AECOPD become more frequent as COPD progresses and have a high recurrence risk within eight weeks. The in-hospital mortality rate for AECOPD may range from $2.5 \%$ to $25 \%$; readmission rates may be from $25 \%$ to $55 \%$ for those who survived, and $25 \%$ to $50 \%$ of these patients may die within one year [4]. The single most crucial parameter to determine the risk of mortality in patients experiencing AECOPD is the forced expiratory volume in one second (FEV1). Other risks include hypoxemia or hypercapnia upon arterial blood gas (ABG) analysis, short distance walked in a fixed time, the severity of functional dyspnea, and low body mass index [1].

There is extensive literature available on the prognostic indices for stable COPD, and instruments have been standardized, after thorough clinical investigations, for predicting the risk of mortality. One such score is the BODE index (i.e., body mass index, airflow obstruction, dyspnea, and exercise) [1]. However, the data on prognostic instruments in settings of AECOPD are not well-established. Steer et al. in 2010 studied this potential gap in the scientific data and noted that individual predictors of mortality were robust enough to be standardized for AECOPD [4]. Steer later developed a comprehensive score to predict the risk of in-hospital mortality in AECOPD called the DECAF score [3]. DECAF consists of five parameters: dyspnea (D), eosinopenia (E), consolidation (C), acidemia (A), and atrial fibrillation (F). DECAF is a simple tool that can be administered at the bedside using indices routinely available on admission. Administering DECAF in severely ill patients can help to predict mortality. Identified high-risk patients must be closely monitored with efficient and timely medical interventions to help reduce the overall mortality rate. We conducted this study to evaluate the DECAF score as a clinical prediction of mortality for patients with AECOPD.

\section{Materials And Methods}

We conducted this prospective study at the intensive care unit (ICU) of the Department of Pulmonology, Civil Hospital, Jamshoro, from January 2016 to December 2018. The study design was approved by the institutional review board. The study included patients aged 35 years or older who were admitted to the ICU and had a primary clinical diagnosis of AECOPD, spirometry consistent with airflow obstruction (FEV1/forced vital capacity $<0.70$ ), and a smoking history of $\geqslant 10$ cigarette packs per year. Patients excluded from the study were those with domiciliary ventilation, survival-limiting comorbidity (e.g., metastatic malignancy), or those whose primary reason for admission was something other than AECOPD.

Sociodemographic data including age, gender, comorbidities, housebound status, and number of previous AECOPD were collected on admission. Clinical data were also collected on admission and consisted of plain chest x-ray, spirometry, electrocardiogram, ABG analysis, complete blood count, kidney function test, liver function test, and serum electrolytes. A DECAF Score [1] was calculated for each patient according to Table 1. 


\section{Cureus}

\section{Variables (DECAF)}

Dyspnea

eMRCD 5 a (too breathless to leave the house unassisted but independent in washing and/or dressing)

eMRCD 5b (too breathless to leave the house unassisted and requires help with washing and dressing)

Eosinopenia (eosinophils $<0.05 \times 10^{9} / \mathrm{L}$ )

Consolidation

Moderate or severe acidemia $(\mathrm{pH}<7.3)$

Atrial fibrillation (including history of paroxysmal atrial fibrillation)

\section{TABLE 1: DECAF score calculation}

DECAF: dyspnea, eosinopenia, consolidation, acidemia, and atrial fibrillation; eMRCD, extended Medical Research Council dyspnea score [2].

Data were analyzed using IBM SPSS for Windows, Version 19.0 (IBM Corp, Armonk, NY). We compared survivors to non-survivors. Mean and standard deviation was calculated for continuous variables. The frequency of the sociodemographic and clinical characteristics of both study groups were compared; chi-square was applied. $\mathrm{P}<0.05$ was considered significant. The frequency of each score for survivors and non-survivors were calculated and compared.

\section{Results}

A total of 162 patients were included in the study. The mean age of these patients was $69 \pm 5$ years. There were 93 (57.4\%) men and 69 (42.5\%) women. There were 65 (40.1\%) participants who were housebound due to the severity of their disease. The mean duration of hospital stay was $4 \pm 3$ days. In total, 141 patients (87.0\%) survived the AECOPD, were successfully managed, and discharged to home. Twenty-one patients (13.0\%) died in the hospital.

We compared the admission characteristics of survivors and non-survivors. We noted that men were more common in both groups. Non-survivors were more likely to be housebound and on long-term oxygen therapy. Non-survivors were also older and had lower mean FEV1 scores. The differences were statistically significant as shown in Table 2. 


\section{Cureus}

\begin{tabular}{llll}
\hline Patient characteristics at the time of admission & Survivors $(\mathbf{n = 1 4 1 )}$ & Non-survivors (n=21) & P-value \\
\hline Gender (male/female) & $82(58.2 \%) / 59(41.8 \%)$ & $11(52.3 \%) / 10(47.6 \%)$ & 0.000 \\
Housebound & $46(32.6 \%)$ & $19(90.47 \%)$ & 0.0006 \\
Age in years (mean \pm SD) & $66.5 \pm 7.23$ & $71.3 \pm 8.48$ & 0.26 \\
Number of previous AECOPD (mean \pm SD) & $3.0 \pm 0.71$ & $3.2 \pm 1.01$ & 0.04 \\
FEV 1 (mean \pm SD) & $43 \pm 12.71$ & $39 \pm 13.11$ & 0.0008 \\
Long-term oxygen therapy (\%) & $19(13.47 \%)$ & $9(42.8 \%)$ & \\
eMRCD, median (range) & $4(3-5 b)$ & $5(5 a-5 b)$ & \\
Co-morbidity status & & & 0.96 \\
Stroke & $13(9.21 \%)$ & $2(9.52 \%)$ & 0.85 \\
Ischemic heart disease & $50(35.46 \%)$ & $7(33.33 \%)$ & 0.98 \\
Heart & $60(42.55 \%)$ & $9(42.85 \%)$ & 0.75 \\
Diabetes & $24(17.02 \%)$ & $3(14.28 \%)$ & 0.0001 \\
Atrial fibrillation & $15(10.6 \%)$ & $9(42.85 \%)$ & 0.43 \\
Renal comorbidities & $18(12.76 \%)$ & $4(19.04 \%)$ &
\end{tabular}

\section{TABLE 2: Comparison of the characteristics of survivors and non-survivors at the}

time of admission

SD, standard deviation; AECOPD, acute exacerbations of chronic obstructive pulmonary disease; FEV1, forced expiratory volume in one second; eMRCD, extended Medical Research Council dyspnea score.

A comparison of the clinical and radiological characteristics of survivors and non-survivors is presented in Table 3. In the survivors group, patients were more frequently seen to have purulent sputum and were less likely to have ineffective cough. Acute confusion and consolidation on chest $\mathrm{x}$-ray was more commonly seen in non-survivors. We witnessed that non-survivors had a higher mean respiratory rate and mean partial pressure of carbon dioxide. The differences were statistically significant as shown in Table 3. 


\section{Cureus}

\begin{tabular}{|c|c|c|c|}
\hline Clinical and radiological characteristics & Survivors $(n=141)$ & Non-survivors $(n=21)$ & P-value \\
\hline Purulent sputum & $93(65.9 \%)$ & $7(33.3 \%)$ & 0.006 \\
\hline Ineffective cough & $18(12.7 \%)$ & $9(42.8 \%)$ & 0.002 \\
\hline Acute confusion & $15(10.6 \%)$ & $10(47.6 \%)$ & 0.00015 \\
\hline Heart rate/min* & $101.3 \pm 20.2$ & $102.1 \pm 21.6$ & 0.61 \\
\hline Systolic blood pressure* & $141.5 \pm 27.21$ & $137.7 \pm 26.12$ & 0.55 \\
\hline Diastolic blood pressure ${ }^{*}$ & $78.3 \pm 16.8$ & $76.4 \pm 17.8$ & 0.63 \\
\hline Respiratory rate/min* & $24.5 \pm 5.24$ & $28.1 \pm 6.2$ & 0.0047 \\
\hline Temperature (Celsius)* & $37.0 \pm 0.56$ & $36.89 \pm 0.51$ & 0.40 \\
\hline Body mass index $\left(\mathrm{kg} / \mathrm{m}^{2}\right)^{*}$ & $24.23 \pm 5.2$ & $22.81 \pm 6.1$ & 0.25 \\
\hline Radiological consolidation & 49 (34.7\%) & $16(76.2 \%)$ & 0.0005 \\
\hline \multicolumn{4}{|l|}{ Arterial blood gases analysis } \\
\hline $\mathrm{pH}^{*}$ & $7.42 \pm 0.09$ & $7.31 \pm 0.06$ & $<0.0001$ \\
\hline $\mathrm{paO}_{2}(\mathrm{~mm} \mathrm{Hg})^{*}$ & $66.23 \pm 10.2$ & $64.12 \pm 11.12$ & 0.38 \\
\hline $\mathrm{paCO}_{2}(\mathrm{~mm} \mathrm{Hg})^{*}$ & $42.52 \pm 7.1$ & $49.81 \pm 12.46$ & 0.0001 \\
\hline $\mathrm{HCO}_{3}(\mathrm{~mm} \mathrm{Hg})^{*}$ & $28.23 \pm 5.6$ & $27.81 \pm 6.1$ & 0.75 \\
\hline Oxygen satur & $92.12 \pm 5.2$ & $92.23 \pm 5.3$ & 0.9 \\
\hline
\end{tabular}

\section{TABLE 3: Comparison of the clinical and radiological characteristics of survivors and non-survivors at the time of admission}

*Mean and standard deviation calculated.

paO2, partial pressure of oxygen; paCO2, partial pressure of carbon dioxide; $\mathrm{HCO}$, hydrogen bicarbonate.

We calculated DECAF scores for all patients, and a DECAF score of three was most common $(n=44 ; 27.2 \%)$. The rate of mortality was $13 \%(n=21)$. On DECAF score zero, $2.9 \%$ of patients died, and 97.1\% survived ( $\mathrm{p}=0.04$ ). On DECAF score one, $2.6 \%$ of patients died and $97.3 \%$ survived ( $\mathrm{p}=0.03$ ). On DECAF scores two and three, $9.7 \%$ and $15.9 \%$ of patients died, respectively, and $90.3 \%$ and $84.1 \%$ survived, respectively. The difference was not statistically significant. On DECAF scores four and five, $60 \%$ and $75 \%$ patients died, respectively, and $40 \%$ and $25 \%$ survived, respectively $(\mathrm{p}<0.00001 ; \mathrm{p}=0.0001)$. No patients had a DECAF score of six

(Table 4). 


\section{Cureus}

\begin{tabular}{|c|c|c|c|c|}
\hline DECAF Score & Total study sample $(\mathrm{N}=162)$ & Non-survivors $(\mathrm{N}=\mathbf{2 1})$ & Survivors (N=141) & $P$ value \\
\hline 0 & $35(21.6 \%)$ & $1(2.9 \%)$ & $34(97.1 \%)$ & 0.04 \\
\hline 1 & $38(23.4 \%)$ & $1(2.6 \%)$ & $37(97.3 \%)$ & 0.03 \\
\hline 2 & $31(19.1 \%)$ & $3(9.7 \%)$ & $28(90.3 \%)$ & 0.54 \\
\hline 3 & $44(27.2 \%)$ & $7(15.9 \%)$ & $37(84.1 \%)$ & 0.49 \\
\hline 4 & $10(6.2 \%)$ & $6(60.0 \%)$ & $4(40.0 \%)$ & $<0.00001$ \\
\hline 5 & $4(2.5 \%)$ & $3(75.0 \%)$ & $1(25.0 \%)$ & 0.0001 \\
\hline 6 & $0(0 \%)$ & $0(0 \%)$ & $0(0 \%)$ & \\
\hline
\end{tabular}

TABLE 4: Comparison of the DECAF scores of survivors and non-survivors

DECAF: dyspnea, eosinopenia, consolidation, acidemia, and atrial fibrillation

\section{Discussion}

COPD is among the more common and debilitating non-communicable lung diseases. The disease is even more severe in middle- and low-income countries due to respiratory exposures associated with poverty and lack of hygiene, restricted access to healthcare, inadequate knowledge about the health hazards of smoking, and difficulty seeking medical care due to various reasons including lack of funds and health insurance [5]. In such settings, it becomes necessary to customize investigation and treatment modalities to make them cheaper, easily accessible, and readily administrable. The DECAF score system is a predictor of in-hospital mortality associated with AECOPD which uses clinical, serological, and radiological parameters. According to this study, DECAF scores of 0-1 are strong predictors of survival, and DECAF scores of 4-6 are strong predictors of mortality.

The rate of mortality in this study was $13 \%$ which was comparable to other studies which have reported a mortality rate of $10.4 \%$ [3], 7.7\% [6], and 12.5\% [7]. In a comprehensive analysis of patients with AECOPD, the highest mortality was seen in patients with DECAF scores of 3-5 (92\%). The in-hospital mortality rate increased with every point increase in the DECAF score [8]. In another study with an $18 \%$ mortality rate in patients with AECOPD, patients who died scored higher on both DECAF and the BAP-65 metric (B: elevated blood urea nitrogen, A: altered mental status, P: pulse $>109,65$ : age $>65$ years). The sensitivity of the DECAF score and BAP-65 score for predicting mortality was $100 \%$, and specificity was $34.1 \%$ and $63.4 \%$, respectively [9]. However, a detailed comparison of the two scales suggests that DECAF is superior to BAP-65 [10]. In another study, most of the patients with 30-day mortality scored four on DECAF; a higher DECAF score was significantly associated with the 30-day mortality score [11]. The DECAF score has also been used to gauge the length of hospital stay in patients with AECOPD; as the DECAF score increased, the length of hospital stay also increased [12-13].

The DECAF score is a clinical, serological, and radiological scale which can be effectively utilized in predicting mortality risk in patients with acute exacerbation of COPD. The widespread use of the DECAF score in pulmonology can help identify high-risk patients at the level of emergency room triage. Urgent medical interventions and intensive care for the patients identified through DECAF can help reduce AECOPD mortality. 
Our study was limited in that we did not assess the relationship between the DECAF score and length of hospital stay. Additional future DECAF studies with robust methodologies and larger samples including multiple tertiary care centers across Pakistan are warranted.

\section{Conclusions}

DECAF is a simple tool that predicts mortality that incorporates routinely available indices. It effectively stratifies COPD patients admitted with acute exacerbations into mortality risk categories. DECAF scores of zero to one are strong predictors for survival, and DECAF scores of four to six are strong predictors of mortality. The DECAF system can facilitate the efficient management of patients with AECOPD.

\section{Additional Information Disclosures}

Human subjects: Consent was obtained by all participants in this study. Liaquat University of Medical and Health Sciences issued approval 29/EC/O/16. Animal subjects: All authors have confirmed that this study did not involve animal subjects or tissue. Conflicts of interest: In compliance with the ICMJE uniform disclosure form, all authors declare the following:

Payment/services info: All authors have declared that no financial support was received from any organization for the submitted work. Financial relationships: All authors have declared that they have no financial relationships at present or within the previous three years with any organizations that might have an interest in the submitted work. Other relationships: All authors have declared that there are no other relationships or activities that could appear to have influenced the submitted work.

\section{References}

1. Celli BR, Cote CG, Marin JM, et al.: The body-mass index, airflow obstruction, dyspnea, and exercise capacity index in chronic obstructive pulmonary disease. N Engl J Med. 2004, 350:1005-1012. 10.1056/NEJMoa021322

2. Viniol C, Vogelmeier CF: Exacerbations of COPD. Eu Respir Rev. 2018, 27:170103. 10.1183/16000617.0103-2017

3. Steer J, Gibson GJ, Bourke SC: The DECAF score: predicting hospital mortality in exacerbations of chronic obstructive pulmonary disease. Thorax. 2012, 67:970-976. 10.1136/thoraxjnl-2012-202103

4. Steer J, Gibson GJ, Bourke SC: Predicting outcomes following hospitalization for acute exacerbations of COPD. Int J Med. 2010, 103:817-829. 10.1093/qjmed/hcq126

5. Lowe KE, Make BJ, Crapo JD, et al.: Association of low income with pulmonary disease progression in smokers with and without chronic obstructive pulmonary disease. ERJ Open Res. 2018, 4:00069-2018. 10.1183/23120541.00069-2018

6. Echevarria C, Steer J, Heslop-Marshall K, et al.: Validation of the DECAF score to predict hospital mortality in acute exacerbations of COPD. Thorax. 2016, 71:133-140.

10.1136/thoraxjnl-2015-207775

7. Nafae R, Embarak S, Gad DM: Value of the DECAF score in predicting hospital mortality in patients with acute exacerbation of chronic obstructive pulmonary disease admitted to Zagazig University Hospitals, Egypt. Egypt J Chest Dis Tuberc. 2015, 64:35-40. 10.1016/j.ejcdt.2014.10.007

8. Collier L, David T, Craig C, Yadavilli R: P45 practical use of the DECAF score: can we improve outcomes in acute exacerbation of COPD admissions?. BMJ Open Respir Res. 2018, 73:A98. 10.1136/thoraxjnl-2015-207770.182

9. Sangwan V, Chaudhry D, Malik R: Dyspnea, eosinopenia, consolidation, acidemia and atrial fibrillation score and BAP-65 score, tools for prediction of mortality in acute exacerbations of chronic obstructive pulmonary disease: a comparative pilot study. Ind J Crit Care Med. 2017, 21:671-677. 10.4103/ijccm.IJCCM_148_17

10. Echevarria C, Steer J, Bourke SC: The DECAF score is a superior predictor of in-hospital death 


\section{Cureus}

than the BAP-65 score. J Clin Respir Dis Care. 2016, 2:1000111. 10.4172/2472-1247.1000111

11. Shafuddin E, Chang CL, Hancox RJ: Comparing severity scores in exacerbations of chronic obstructive pulmonary disease. Clin Respir J. 2018, 12:2668-2675. 10.1111/crj.12973

12. Ahmed M, Faryal S, Sheikh K: Complex DECAF scores prolong the hospital stay of patients suffering from COPD. Int J Endorsing Health Sci Res. 2017, 5:52-55.

13. Yadavilli RK, Shah N, Craig C, Collier L: Does higher DECAF score increase length of hospital stay in acute exacerbation of COPD?. Eu Respir J. 2016, 48:PA3437.

10.1183/13993003.congress-2016.PA3437 